\title{
FACTORES QUE AFECTAN EL \\ FUNCIONAMIENTO DE INSTALACIONES \\ FOTOVOLTAICAS AUTONOMAS EN \\ REGIONES DEL NORESTE ARGENTINO
}

Dr. A. Busso, Dr. L. Vera, Dr. M. Cáceres, Ing. A. Firman, Tco. R. Sánchez (*)

RESUMEN: En un contexto actual de gran expansión de los sistemas de generación fotovoltaica, las experiencias de terreno en aplicaciones para el sector rural son limitadas y se hace urgente comenzar a analizar en detalle los factores que más afectan el funcionamiento de tales sistemas. Tomando como base, datos de mantenimiento correspondientes a 85 sistemas fotovoltaicos autónomos (SFA) instalados en escuelas rurales de la provincia de $\mathrm{Co}^{-}$ rrientes, se realizó un estudio de los principales problemas registrados durante los dos primeros años de operación y de sus causas. Como elemento primordial surge la calidad del diseño inicial del SFA, comprobándose que para Corrientes no se seleccionaron elementos adaptados a las condiciones reales de la zona. Otro factor clave, y no menos importante para lograr un grado de confiabilidad aceptable, es respetar una buena planificación en el mantenimiento tal como se ha verificado con la experiencia acumulada en Corrientes.

Palabras clave: sistemas fotovoltaicos autónomos, electrificación rural descentralizada.

\section{INTRODUCCIÓN}

Durante los últimos años, el mercado del solar fotovoltaico creció de manera acelerada, siendo el sector del fotovoltaico conectado a red el más dinámico (REN21, 2010). El motivo de esta explosión se debe esencialmente a la implementación de programas nacionales en países desarrollados que favorecen las "energías limpias", en un contexto actual de preocupación respecto al calentamiento global y el agotamiento de los recursos energéticos tradicionales.

Aprovechando esta dinámica, los nuevos proyectos en electrificación rural en los países en desarrollo se dedican principalmente a la instalación de sistemas fotovoltaicos autónomos (SFA). Argentina sigue esta tendencia y a través del Proyecto de Energías Renovables en Mercados Rurales (PERMER), numerosas provincias ya se beneficiaron de nuevas instalaciones fotovoltaicas para abastecer de energía eléctrica a escuelas, centros comunitarios, puestos sanitarios y viviendas. Se trata de un programa financiado por el Gobierno Nacional, donde el 70\% es aportado por la Secreta- 
ría de Energía a través de un préstamo del Banco Mundial (BM).

Si bien los SFA representan un porcentaje bajo en la potencia eléctrica generada en Argentina con fuentes renovables de energía, un $0,02 \%$ según datos informados por el Observer del año 2006, son importantes respecto a la proporción de la población que no dispone de un servicio convencional de energía eléctrica y se ve beneficiada por esta tecnología. Dentro de este contexto, actualmente, unas 70000 familias argentinas no cuentan con acceso a la energía eléctrica (United Nations, 2009). El programa PERMER, a nivel país instalo 1369 SFA en escuelas y más de 4300 SFA para viviendas familiares, lo que representa una generación aproximada de $1 \mathrm{MWp}$ (Red REEEP, 2009). A estos últimos datos se les debe sumar el aporte de instalaciones realizadas a través de iniciativas privadas o de otros programas de electrificación rural. De esta forma, se estima que para el año 2006, 7000 hogares familiares recibían electricidad vía generación fotovoltaica (Scarabée, 2008). Además, el PERMER prevé instalar a corto plazo 15690 nuevos SFA en 12 provincias del país, lo que representa una potencia de alrededor a los 1,9 MWp (PERMER, 2009).

A comparación de los sistemas fotovoltaicos conectados a una red de distribución, los SFA se implementan generalmente cómo fuente única de energía, donde uno de los puntos clave es el almacenamiento de esta energía, y deben cubrir por sí solos la demanda energética para la que han sido diseñados. Básicamente, un sistema fotovoltaico autónomo se constituye de un panel fotovoltaico o grupo de módulos que convierte la energía solar en energía eléctrica, un regulador de carga que controla la conexión entre los paneles, la carga y las baterías de forma que esta última no se descargue ni se sobrecargue en exceso, baterías para almacenar la energía generada por los módulos fotovoltaicos, y a veces de un inversor que convierte la energía continua que generan los paneles en la energía alterna que necesite el equipo. Cuando el uso se limita a iluminación se eligen sistemas solamente de corriente continua por una cuestión de simplicidad, pero, generalmente, resulta necesaria una conversión a corriente alterna debido a que la mayoría de los artefactos utilizados funcionan con este tipo de corriente. (Labouret A. y Volloz M., 2008). Para el adecuado dimensionamiento de un SFA existen métodos de cálculo basados en estimaciones de consumo diario y de datos meteorológicos disponibles. No obstante, la configuración del sistema resulta un proceso más complejo donde se deben considerar todas las interacciones entre cada elemento del SFA para entender los eventuales errores de dimensionamiento y de configuración que se observan en algunos proyectos.

Si en teoría estos sistemas son confiables, las particularidades de cada región difieren y la integración de SFA en zonas muy aisladas es todavía algo nuevo, o sea, no se conocen a ciencia cierta las expectativas de generación a corto y largo plazo de la mayoría de los SFA instalados en el país, ya que son muy pocos los estudios sobre SFA en actividad. De esta situación surgen dos necesidades: la de contar con un registro actualizado de las instalaciones fotovoltaicas en la región, y la de establecer un intercambio de información entre equipos técnicos para facilitar la capacitación del personal involucrado en tales proyectos.

Dependiendo de la provincia, el mantenimiento de los SFA instalados a través del PERMER es realizado por diferentes actores sociales. Para la provincia de 
Corrientes, en la que los SFA se encuentran en establecimientos escolares, la responsabilidad es del Ministerio de Educación y Cultura. Este organismo, a través de un convenio, encomendó al Grupo Energías Renovables (GER - FaCENA - UNNE) las tareas de mantenimiento de estos SFA teniéndose, de esta manera, acceso a una información de primera mano, útil para el estudio del comportamiento de estos sistemas en condiciones reales de uso.

En este artículo se presentan las primeras observaciones sobre el funcionamiento de 85 SFA obtenidas en la provincia de Corrientes. Se presenta un análisis de los principales problemas registrados y de los factores que influyen sobre la sostenibilidad de los SFA en la región. Los datos obtenidos de provincias vecinas permiten además visualizar las dificultades que aparecen en el momento de plantear un mantenimiento apropiado con el objetivo de realizar un intercambio de experiencias dentro del país.

\section{METODOLOGÍA Y DATOS DIS- PONIBLES}

Por cuestiones de disponibilidad e integridad de la información, el presente estudio se centra esencialmente en los SFA instalados a través del PERMER en la provincia de Corrientes. Los SFA estudiados son relativamente jóvenes, entraron en operación durante el año 2008 y tienen, al momento del presente estudio, entre un año y medio y dos años de funcionamiento. Durante este lapso de operación, aparecieron diversos problemas, algunos suficientes graves para dejar en la actualidad a un 10\% de las escuelas sin energía.

Los factores que pueden afectar al funcionamiento de un SFA en zonas rurales aisladas son múltiples. En general, los proyectos de electrificación descentralizada toman en cuenta aspectos técnicos, considerando los ámbitos económicos, sociales y ambientales, en una política denominada de desarrollo sostenible o sustentable, donde se busca principalmente mejorar la tecnología para minimizar el impacto de actividades humanas sobre el medio ambiente.

De manera inversa, factores sociales o ambientales pueden afectar al funcionamiento de sistemas tecnológicos y en el caso particular de los SFA se observa tanto una relación directa con los usuarios, cómo ubicaciones en zonas geográficas austeras, que pueden resultar ser factores predominantes en el funcionamiento de los sistemas. En consecuencia, hablar de sostenibilidad de los SFA en el tiempo se entiende como una necesaria capacidad de los sistemas de realizar su función de la manera prevista, o sea, en condiciones reales de operación y por un periodo de vida mínimo.

Para tales sistemas complejos, constituidos de varios elementos, se suele hablar también de fiabilidad, o grado de confiabilidad, tema en un principio asociado a la seguridad de una instalación (una central nuclear por ejemplo), pero en cuanto a los sistemas fotovoltaicos, se realizan estudios de fallos, más relacionados con la indisponibilidad del sistema y, básicamente, por su efecto sobre el costo económico derivado. Hasta ahora, los estudios de fiabilidad de SFA se han centrado fundamentalmente en las labores de dimensionamiento, sin considerar la evolución del sistema en operación real, la degradación y averías de sus componentes y por tanto, su influencia sobre la disponibilidad energética inicialmente estimada (Villar, 2003).

En Corrientes, datos disponibles a los dos años de las primeras instalaciones pueden permitir una evaluación de la fia- 
bilidad de los SFA instalados. Desde el inicio del programa, el GER realizó las principales intervenciones de mantenimiento $\sin$ que se haya podido seguir un ritmo
"Total de referencia", figuran 50 visitas sobre un total de 43 escuelas indicando que algunas escuelas fueron visitadas dos veces, fin del 2009 y mediados del 2010.

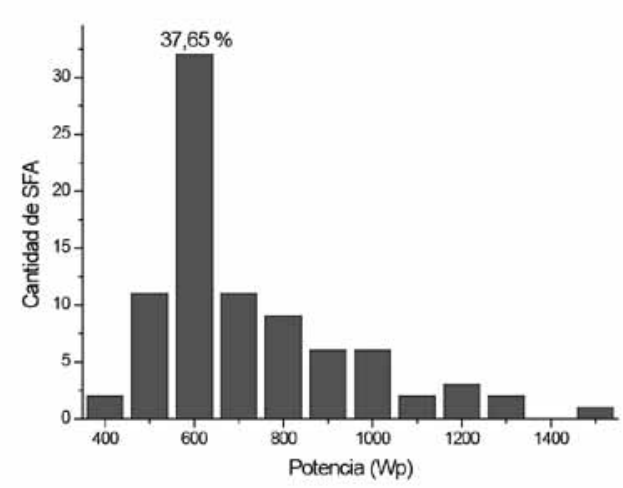

Figura 1: Distribución de las potencias instaladas en los SFA de Corrientes

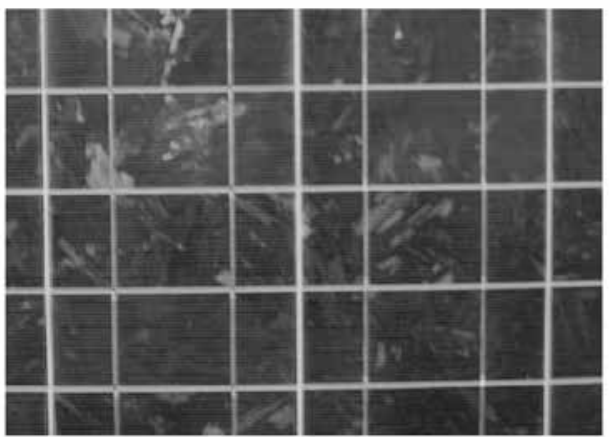

Figura 2: Decoloración observada en módulos FV. continuo de visitas por diversas cuestiones relacionadas, principalmente, a la falta de recursos económicos destinados a tales actividades. A partir del mes de Marzo 2010, se implemento un cronograma de visitas con operaciones sistemáticas de mantenimiento con el compromiso de controlar por lo menos 2 veces por año cada sistema, esto representa unas 16 visitas mensuales a realizar. Los datos de mantenimiento obtenidos a partir de esta fecha son los más relevantes para establecer estadísticas, suman 50 intervenciones, con 43 esuelas visitadas (el 50\% de los SFA instalados) y al concentrarse en pocos meses, representan un estado general de las escuelas a mitad del año 2010. La figura 1 muestra la distribución por potencia de los sistemas instalados en las escuelas de la provincia y en la Tabla 1 se detalla la cantidad total instalada en las mismas por tipo de equipamiento. En la Tabla 3 se exponen los problemas mas frecuentes encontrados durante las visitas realizadas en el 2010. En ella se observa que en la primer columna

\section{Problemas encontrados según compo- nente de la instalación}

Baterias: Un problema común al $74 \%$ de los SFA controlados fue la falta de líquido en los vasos de las baterías utilizadas (tipo plomo-acido, de 220Ah), inclusive en escuelas visitadas el año anterior (Tabla 2). Mantener un nivel permanente de electrolito es indispensable para asegurar un tiempo de vida razonable de las baterías. En todas las intervenciones se completaron, cuando el caso lo requería, los vasos "seco" con agua destilada, sin garantía alguna de que recuperen su nivel óptimo de carga. En algunos casos se debió agregar hasta $700 \mathrm{~cm} 3$ de agua destilada por vaso.

Se constató también una disparidad de tensiones entre baterías de un mismo banco. La asociación de baterías con diferentes características implica que algunas se sobrecargan cuando otras nunca llegan a la tensión esperada, lo que provoca la degradación de las baterías en buen estado funcional. Por otro lado, la merma de autonomía exhibida durante las horas sin sol, relativa al 
Tabla 1: Detalle de los elementos que constituyen los SFA de Corrientes.

\begin{tabular}{|l|l|l|c|}
\hline Elemento & Marca & Características & Cantidad \\
\hline Módulo Fotovoltaico & PHOTON - modelo PM0050 & $\begin{array}{l}12 \mathrm{~V}-50 \mathrm{Wp} \text { (potencia } \\
\text { nominal) }\end{array}$ & 1234 \\
\hline $\begin{array}{l}\text { Acumulador (batería plomo- } \\
\text { acido) }\end{array}$ & $\begin{array}{l}\text { AUTOBAT - modelo I-12-18 } \\
\text { Solar }\end{array}$ & $\begin{array}{l}12 \mathrm{~V}-220 \mathrm{Ah} \text { (capacidad } \\
\text { nominal) }\end{array}$ & 430 \\
\hline Regulador de carga & $\begin{array}{l}\text { CONERGY - modelo SCC20 } \\
\text { eco }\end{array}$ & $\begin{array}{l}12 / 24 \mathrm{~V}-20 \mathrm{~A} \text { (corriente } \\
\text { máxima) }\end{array}$ & 41 \\
\hline Regulador de carga & $\begin{array}{l}\text { CONERGY - Modelo SCC40 } \\
\text { vision }\end{array}$ & $\begin{array}{l}12 / 24 \mathrm{~V}-40 \mathrm{~A} \text { (corriente } \\
\text { máxima) }\end{array}$ & 64 \\
\hline Inversor CC/CA & STUDER - modelo AJ 2400 & $24 \mathrm{~V}-2000 \mathrm{VA}$ (P nominal) & 46 \\
\hline Inversor CC/CA & STUDER - modelo SI 2324 & $24 \mathrm{~V}-2300 \mathrm{VA}$ (P nominal) & 64 \\
\hline Inversor CC/CA & STUDER - modelo SI 3324 & $24 \mathrm{~V}-3300 \mathrm{VA}$ (P nominal) & 30 \\
\hline
\end{tabular}

Tabla 2: Problemas más frecuentes encontrados en los SFA de Ctes, (6 primeros meses del año 2010).

\begin{tabular}{|c|c|c|c|c|c|}
\hline $\begin{array}{c}\text { Total } \\
\text { de referencia }\end{array}$ & $\begin{array}{c}\text { Escuelas que } \\
\text { presentan } \\
\text { baterías con } \\
\text { vasos "seco" }\end{array}$ & $\begin{array}{c}\text { Escuelas con un } \\
\text { inversor fuera } \\
\text { de servicio }\end{array}$ & $\begin{array}{c}\text { Escuelas con un } \\
\text { regulador fuera } \\
\text { de servicio }\end{array}$ & $\begin{array}{c}\text { Escuela } \\
\text { actualmente } \\
\text { sin energía }\end{array}$ & $\begin{array}{c}\text { Robo parcial } \\
\text { o total de SFA }\end{array}$ \\
\hline Cantidad & 32 & 8 & 1 & 10 & 4 \\
\hline 50 visitas & $64 \%$ & $16 \%$ & $2 \%$ & $20 \%$ & $8 \%$ \\
\hline 43 escuelas & $74 \%$ & $19 \%$ & $2 \%$ & $23 \%$ & $9 \%$ \\
\hline
\end{tabular}

nivel original durante los primeros meses de operación, según lo manifestado por el personal docente de las escuelas afectadas, muestra una disminución notable de la capacidad de almacenamiento de estos SFA.

Como observación adicional se debe mencionar que se detectaron algunos casos (mínimos) de bornes sulfatados, lo que generaba un estado de alerta del regulador de carga.

Se constato también una disparidad de tensiones entre baterías de un mismo banco. La asociación de baterías con diferentes características implica que algunas se sobrecargan cuando otras nunca llegan a la tensión esperada, lo que provoca la degradación de las baterías en buen estado funcional. Por otro lado, la merma de autonomía exhibida durante las horas sin sol, relativa al nivel original durante los primeros meses de operación, según lo manifestado por el personal docente de las escuelas afectadas, muestra una disminución notable de la capacidad de almacenamiento de estos SFA.
Como observación adicional se debe mencionar que se detectaron algunos casos (mínimos) de bornes sulfatados, lo que generaba un estado de alerta del regulador de carga.

Inversores: En el año 2009, se cambiaron 6 inversores en diferentes escuelas y durante los cuatro meses registrados de mantenimiento para el año 2010, se retiraron 7 de los 8 inversores afuera de servicio: 3 del modelo SI 2324 y 4 del modelo SI 3324. Se observó que el factor predominante de la salida de servicio de inversores fue la presencia de ranas o insectos. Otra observación fue que en varios informes registrados en escuelas que sufrieron la salida de servicio de un inversor, se sospecha como causa probable la conexión de aparatos defectuosos (bomba de agua principalmente).

Reguladores: Desde la puesta en operación de los SFA solamente dos reguladores de carga presentaron fallas sin que se puedan identificar las causas exactas. En uno de los casos, el sobrecalentamiento produjo el derretimiento de la carcasa del regulador 
sin la activación de los elementos de protección. Si bien no hubo incendio, es un riesgo existente a tomar en cuenta. No obstante ello las casillas cuentan con su extinguidor para casos de urgencia.

Módulos: En dos años de operación, se ha comprobado una decoloración con un patrón característico en algunos módulos. La misma se produce a lo largo de la zona central de las celdas tal como se aprecia en la figura 2. No se tiene todavía un registro completo de los SFA afectados ni la causa probable a la que obedece este fenómeno, presumiéndose que se debe a una pérdida de la capa anti-reflectante del panel.

A modo de comparación, se recabo información sobre mantenimiento y desperfectos en SFA instalados en otras provincias. En el caso de la provincia del Chaco por ejemplo, los sistemas instalados son tanto domiciliarios como destinados a escuelas. Cada provincia presenta también diferencias en lo que respecta al clima: en el Chaco, la gran mayoría de las instalaciones se encuentran en el Impenetrable Chaqueño, región donde las temperaturas anuales registradas son casi las más altas del país. Este motivo provoco la necesidad de reemplazar de una gran parte de los reguladores de carga de los SFA domiciliarios. La gestión de los SFA se hace a través de la Subsecretaria de Energía y Programas Especiales de la Provincia del Chaco, la cual contrató a la Cooperativa de Servicios Públicos Norte Chaqueño de J. J. Castelli para el mantenimiento de las instalaciones, con una preocupación actual centrada en el cambio de baterías por envejecimiento precoz no previsto. El primer SFA se instaló en el año 2005, con unos 650 reclamos registrados para el año 2009 (34 \% de los sistemas).

Salta es otra provincia importante a considerar en estas comparaciones. En ella, la instalación de los primeros SFA a través del PERMER data del año 1998 y a la fecha representan solamente el $39 \%$ de la tota- lidad de los SFA a cargo del Ente Regulador de Servicios Públicos de la Provincia de Salta. En esta provincia, la concesionaria es la Empresa de Servicios Eléctricos Dispersos (ESED S.A.). Según datos aportados por ella, durante los últimos años se registraron un promedio estimativo de 100 reclamos por año, representando el $15 \%$ de los sistemas.

Córdoba, como Salta, presenta una gran cantidad de SFA instalados a través de iniciativas propias del gobierno provincial, de esta forma, de las 230 escuelas con SFA solamente 86 figuran como instaladas a través del PERMER.

\section{DISCUSION DE RESULTADOS}

Como ya se menciono en párrafos anteriores, la provincia de Corrientes viene encarando de manera sistemática desde fines del 2009 el programa de mantenimiento de las escuelas con SFA. Como resultado, a la fecha solo el $10 \%$ de las escuelas en la provincia de Corrientes se encuentran sin energía a causa de fallas en los componentes por diversos motivos.

El caso de Corrientes no es único. En otras provincias, el problema de planificación y financiación del mantenimiento de los SFA que se instalan no esta resuelto, debido a que el grado de confiabilidad de los SFA no resulta ser el que se esperaba y que algunos elementos de los sistemas a mantener tienen un tiempo de vida inferior a lo previsto, o que directamente no son aptos para las condiciones reales de operación. Esto significa que, al momento del diseño conceptual del sistema y/o selección de componentes, no se tomaron en cuenta de manera integral todos los aspectos relacionados con las condiciones geográficas y sociales del lugar de instalación.

Baterias: En Corrientes, según datos proporcionados por encuestas a los maestros de las escuelas que cuentan con un SFA, se observa un envejecimiento precoz de los 
acumuladores. Una de las posibles causas es un uso excesivo del banco de baterías (ciclos de carga y descarga demasiado cortos), tal como muestra la evidencia en un establecimiento donde se encontraron artefactos de alto consumo. A este hecho se debe sumar la falta de mantenimiento adecuado por casi dos años. Los bajos niveles de electrolito registrados en la mayoría de las escuelas, directamente relacionados con la perdida de capacidad de almacenamiento, ponen en evidencia otras causas, como ser falta de visitas regulares para su mantenimiento, mala selección de baterías resultando estas muy sensibles a las altas temperaturas registradas (propias para la región), entre otras. Las prestaciones y características de una batería durante su operación dependen fuertemente de la temperatura de trabajo. Esta variable afecta el voltaje de flote debiendo compensarse para obtener un rendimiento óptimo y tiene consecuencias en la vida útil de la batería. Otro hecho observado fue disparidad en la distribución en las tensiones de batería en un banco dado. Cuando algunas de ellas registraban tensiones superiores a la máxima recomendada $(13,8 \mathrm{~V})$ otras, asociadas en serie o en paralelo exhibían bajas tensiones, $8 \mathrm{~V}$ y menores.

Regulador: A fin de observar el comportamiento general de los componentes instalados en las escuelas, se replico en la terraza del laboratorio del GER, un sistema de 400 $\mathrm{Wp}$, banco acumulador con dos baterías de $75 \mathrm{Ah}$ en serie $(24 \mathrm{~V})$, regulador de $20 \mathrm{~A} \mathrm{e}$ inversor de $2300 \mathrm{~W}$. Se midieron corrientes $\mathrm{y}$ tensiones en diferentes puntos registrándose tensiones de corte entre $28,9 \mathrm{~V}$ y 29,7 $\mathrm{V}$, superiores a los $27,6 \mathrm{~V}$ recomendados, situación esta que puede ser una de las causas de evaporación excesiva del electrolito. Cabe acotar que una característica del regulador de carga empleado es la de no limitar la corriente de carga, llegando esta a $20 \mathrm{~A}$ cuando el panel FV permite entregarlos. A fin de evitar caer nuevamente en este problema, otras provincias optaron baterías de diferente tecnología. Por ejemplo, en la zona de Córdoba, se logró limitar la evaporación de las baterías originales con electrolito líquido remplazándolas por acumuladores de tipo Plomo-Selenio (PB-SE) y en la provincia de Salta, las baterías seleccionadas para los SFA son de tipo Tubulares.

Inversores: Un análisis de la distribución geográfica de las escuelas afectadas en la provincia no permite concluir que una zona sea más sensible que otra. Las ranas encontradas dentro de algunos inversores son del género Scinax, presente en toda la provincia, son de tipo arborícolas (trepadoras) y con hábitos domiciliarios, se las encuentra generalmente en los baños y zonas húmedas de las viviendas. La presencia de ranas se verifico solamente en inversores del modelo SI 3324. Esto se explica porque los forzadores para la ventilación del equipo poseen una rejilla de trama ancha, con espacios libres de $6 \mathrm{~mm}$ contra $3 \mathrm{~mm}$ libres para los otros modelos. Por otro lado, otro aspecto observado como causa de desperfecto, es el hecho que los inversores instalados no vienen de fabrica con la parte posterior de las placas de los circuitos barnizadas, siendo vulnerables a que el ingreso de pequeños insectos, por ejemplo hormigas, causen un corto circuito en los terminales de los transistores de potencia como ha sido el caso en varias escuelas. La importancia del barnizado quedo de manifiesto con un inversor reparado en el año 2009 y que presento problemas nuevamente en el año 2010. E1 barnizado de la parte posterior de las placas durante la primera reparación evitó que el inversor se queme a causa del ingreso de rana. Basto con una simple limpieza para ponerlo en operación nuevamente.

La tecnología electrónica del inversor es también un factor importante respecto a su robustez y se relaciona directamente con la sostenibilidad del SFA. A este respecto, se observa que los inversores de menor potencia, modelos AJ 2400, casi no presen- 
tan problemas registrados a los dos años de operación. Estos modelos son de tipo microcontrolados en comparación con los modelos SI 2324 y SI 3324 que utilizan una electrónica totalmente discreta.

Módulos: La decoloración encontrada en algunos módulos es también un signo de alarma. Una mala calidad de estos productos puede afectar significativamente la vida útil de los SFA. Ante la evidencia de decoloración, varios de estos módulos fueron reemplazados con la finalidad de relevar sus curvas I-V y compararlas con módulos de la misma marca y lote, ensayados en el año 2009 (Bello, 2009), pero en buenas condiciones visuales y funcionales. Los datos obtenidos no muestran diferencia apreciable entre los valores de potencia pico para los dos grupos de módulos ensayados. Se presume que la decoloración puede deberse a una perdida de la capa antirreflectante, efecto que seria observable para grandes ángulos de incidencia de la radiación solar, esto es, durante las horas de la mañana y la tarde. Este punto esta pendiente de ensayo.

Dimensionamiento: Finalmente, un dimensionamiento errado del banco de baterías o del banco de generación puede llevar a perjudicar la vida útil del SFA. Para las 85 escuelas del estudio, este dimensionamiento fue realizado por el Ministerio de Educación, a través de una estimación del consumo de cada establecimiento, tomando en cuenta las dimensiones y uso de cada ambiente de la escuela y si existe o no una vivienda destinada a los docentes. En contraposición, en la provincia del Chaco se tomó en cuenta para el dimensionamiento de los SFA que se instalaron en escuelas, solamente la cantidad de alumnos y docentes, sin discriminar las necesidades específicas de cada establecimiento. De esta manera, el sistema de mayor potencia instalado en en la provincia del Chaco es de $800 \mathrm{Wp}$ para un establecimiento escolar de 120 alumnos, mientras que en Corrientes se tiene un generación de1500 Wp para una escuela con matricula de 98 alumnos. No obstante ello, existen también diferencias notables entre escuelas de una misma provincia. En Corrientes, el método empleado para el cálculo de la potencia del SFA a instalar (el cual no toma en cuenta la matricula) llevó a destinar $1300 \mathrm{Wp}$ tanto para una escuela de 35 alumnos como para otra de 105 alumnos con el riesgo de sobre uso del sistema que conlleva el ultimo caso.

Figura 3: Clasificación de las operaciones de mantenimiento realizadas en los SFA de Corrientes, durante los 6 primeros meses del año 2010.

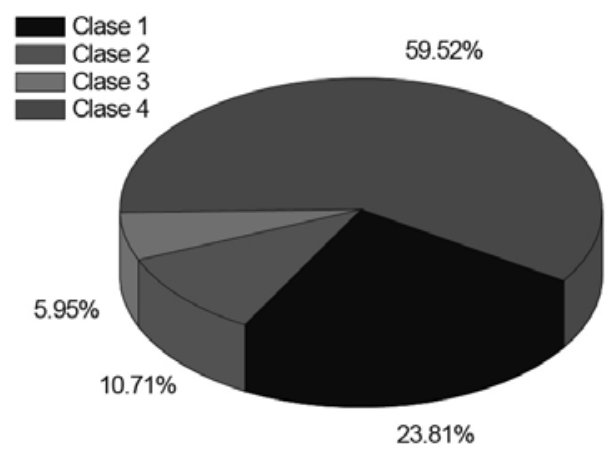

Clase 1: Escuela sin energía, se necesitó intervención especializada (elemento dañado)

Clase 2: $\quad$ Escuela sin energía, la intervención técnica pudo realizarse en el lugar

Clase 3: Escuela sin energía, intervención básica (Ej. reseteo del inversor)

Clase 4: $\quad$ Escuela con energía, visita de control (Ej. se agregó agua en las baterías) 
Organización del mantenimiento: Este es otro factor clave para garantizar una sustentabilidad en el tiempo de estos sistemas. Si bien es necesario controlar el nivel de electrolito de las baterías, estas operaciones no implican necesariamente la intervención de personal especializado. Efectivamente, se observó que en las escuelas donde no se encuentran baterías con vasos "seco", el personal docente o técnico del establecimiento, capacitado previamente durante la fase de puesta en marcha de los sistemas, tomaba la iniciativa de completar regularmente el electrolito con agua destilada. Se debe puntualizar aquí que, a pesar de que la capacitación se llevo a cabo en todas las escuelas, no todo el personal tiene la suficiente destreza y/o predisposición para realizar estas operaciones básicas. Se pueden así diferenciar las visitas de mantenimiento de diferente clase, de acuerdo con la actividad efectuada tal como se aprecia en la figura 3. De esta manera surge, que un 59,5\% de las visitas fueron solamente "de control", lo cual es representativo también de las escuelas que no presentaron problemas graves y se han mantenido con energía desde su puesta en operación.

De forma general, para la provincia de Corrientes, la selección de los diferentes elementos en el diseño de los SFA no resultó ser la mejor solución técnica, ya sea por falta de adaptabilidad a las condiciones reales de operación de la zona y/o por simples cuestiones de calidad. La provisión de fondos vía el Banco Mundial implica de alguna manera entrar en un sistema economicista ("criterio o doctrina que concede a los factores económicos primacía sobre los de cualquier otra índole”, según el Diccionario de la Real Academia Española). Esto explica que al momento de la toma de decisiones frente a diferentes arquitecturas para los SFA, se priorizan los factores económicos por sobre los de confiabilidad y de sustentabilidad. Lógicamente, queriendo ahorrar en la etapa de instalación, se gene- ran problemas técnicos a corto plazo, los cuales quedan a cargo de una entidad local responsable del mantenimiento. Se trata de gastos no previstos en el presupuesto inicial, poniendo en riesgo todo el proyecto y afectando gravemente la imagen de confiabilidad de sistemas alternativos como lo son los SFA para la electrificación rural.

\section{CONCLUSIONES}

A partir de datos de mantenimiento de SFA instalados en escuelas rurales de la provincia de Corrientes y recolectados desde marzo del 2010 a la fecha, se detectaron factores que afectan a la sostenibilidad de estos sistemas de generación. Estos factores no fueron previstos en las etapas de diseño.

Es evidente que las fallas en SFA, actualmente registradas, no deben comprometer la implementación de la energía solar fotovoltaica como solución adecuada a la electrificación rural, si no que se deben aprovechar urgentemente las pocas experiencias en el sector para permitir el sostenimiento en el tiempo de los sistemas instalados y a instalar.

Este punto es de vital interés dado que, en los próximos meses, más de 15000 nuevos SFA serán instalados en 12 provincias argentinas a través del principal programa nacional de promoción de energías renovables. Por ello, es deseable que se tomen en cuenta las características propias a cada región del país, para evitar una generalización de problemas que hoy son conocidos a nivel local pero tal vez sin el necesario retorno de información a nivel nacional.

\section{ABSTRACT}

In a current context of major expansion of photovoltaic generation systems, field experiences in rural applications are limited and it is urgent to start analyzing in detail factors that are affecting the sustainability of such systems. Based on data which characterized 85 stand-alone photovoltaic systems (SPS) insta- 
lled in the province of Corrientes, a study was conducted about major problems encountered during the first two years of operation and its asociated causes. There was highlighted initial design quality of SPS as a major element and ther was found that elements adapted to actual conditions of the area's operation were not correctly selected. Another key factor in the search of an acceptable degree of reliability is a good planification in maintenance.

Keywords: stand-alone photovoltaic systems, decentralized rural electrification.

\section{REFERENCIAS}

C. Bello, V. Jimenez, V. Toranzos, A. Busso, C. Cadena. (2009). Relevador portátil de curvas I-V de paneles fotovoltaicos como herramienta de diagnostico in situ de sistemas de generación fotovoltaica. Avances en Energías Renovables y Medio Ambiente, Vol. 13.

C. Bello, L. H. Vera, A. Busso, C. Cadena. (2009). Proyecto de electrificación rural a través de sistemas fotovoltaicos autónomos en la provincia de Corrientes. Avances en Energías Renovables y Medio Ambiente, Vol. 13.

Labouret A. y Volloz M. (2008). Energie Solaire Photovoltaique. LeMoniteur, Paris.

REN21 (2010). Renewables 2010 Global Status Report. Paris.

Red REEEP (2009). Energías renovables: diagnósticos, barreras y propuestas. Secretaría de Energía y Fundación Bariloche.

Scarabée (2008). Boletín de conexión de la red de expertos en electrificación descentralizada, No 22.

Observ'ER (2007). Worldwide electricity production from renewable energy sources. Ninth inventory.

PERMER (2009). Provisión e instalación de equipos fotovoltaicos e instalación interna en viviendas rurales de diversas provincias. Licitación internacional.

United Nations (2009). Rural Population by Major Area, Region and Country, 1950-2050. 